Table 1

Number of Trials-to-Extinction Yielded by Each $S$ in Each Group

\begin{tabular}{lrrrr}
\hline & \multicolumn{4}{c}{ Groups } \\
& ES & CS & EM & CM \\
\hline Ss & 40 & 40 & 19 & 12 \\
& 40 & 40 & 4 & 6 \\
& 0 & 40 & 11 & 0 \\
& 8 & 40 & 4 & 40 \\
& 40 & 40 & 4 & 13 \\
& 40 & 31 & 3 & 4 \\
& 40 & 40 & 4 & 3 \\
& 5 & 0 & 3 & 0 \\
Totals & 40 & 25 & 5 & 3 \\
\hline
\end{tabular}

of $0.60(p>.20)$. Apparently, the four groups received equivalent durations of punishment during training.

In testing, each $S$ was placed in the start box, oriented goalward, and the guillotine door raised. This action initiated the buzzer. Shock was omitted, of course. Entry into the goal box served to tum on the 3-sec light for all Ss. The S was then conveyed to a holding cage and held there between trials. For the spaced animals, the interval between Trials 1 and 2 was $10 \mathrm{~min}$; for the succeeding nine trials, $5 \mathrm{~min}$ each; and for the final 30 trials, $3 \mathrm{~min}$ each. For the massed $\mathrm{Ss}$, all intertrial intervals were approximately $25 \mathrm{sec}$ in duration.

All Ss were given a total of 40 testing trials or carried to an extinction criterion defined as either a starting laten $y$ (time required to leave the start box) or a running time (time required to negotiate the full length of the alley) in excess of $3 \mathrm{~min}$. A special set of extinction criteria was applied to the initial (only) test trial. If $S$ failed to leave the start box within $3 \mathrm{~min}$, the door was lowered and then raised again 1 min later. If $S$ left the start box within the time limit but failed to traverse the full alley within $3 \mathrm{~min}$, it was returned to the start box, detained there for $1 \mathrm{~min}$, and then given a second opportunity. If $S$ failed to complete the initial run following the third such opportunity, it was retired and zero noted as the number of trials to extinction. Eight animals required this treatment. Four yielded zero runs, the other four, 40, 5, 5, and 3 runs. These eight Ss were not uniquely associated with treatment; three belonged to Group EM, two to CM, two to CS, and one to ES.

\section{RESULTS AND DISCUSSION}

Table 1 presents the number of trials to extinction yielded by each $S$ in each of the four groups. Three conclusions follow readily from inspection of the table: (1) No experimental effect is seen in either the massed or the spaced condition. When intertrial interval is collapsed as a dimension and chi square applied as a test of control and experimental frequencies falling above and below the grand median, a value of but 1.00 is found. (2) A substantial effect is associated with intertrial spacing for both experimental and control treatments. When experimental-control treatment is ignored and chi square applied to the intertrial intervals as a median test, a value of 9.00 is found ( $p<.01$, two-tailed test). (3) No interaction obtains.

This study fails to replicate Murray-Strandberg (1965). Reasons for this contradiction may be sought in the myriad differences between the two experiments in "minor" procedural details, inviting the conclusion that the effect is fragile and/or crucially dependent upon some unidentified parameter. The failure to replicate renders meaningless, of course, the question of whether or not the effect is dependent upon the massing of test trials.

The present finding of greater resistance to extinction in spaced trials is of considerable interest. Related studies are contradictory in outcome. Edmonson \& Amsel (1954) trained rats to escape shock in a nunway and then extinguished them with trials either massed (5 or 15 per day) or spaced ( 1 per day). Greater resistance to extinction was seen in the spaced condition. Hall (1955) found no difference between intertrial extinction intervals of 10 and $90 \mathrm{sec}$ in an avoidance shuttle response. Differential reinforcement best explains the present finding. The spaced Ss enjoyed longer periods of time-out from buzzer-elicited fear at the termination of each run.

\section{REFERENCES}

BECK, R. C. On secondary reinforcement and shock termination. Psychological Bulletin, $1961,58,24-45$.

BINDRA, D. B. Neuropsychological in terpretation of the effects of drive and incentive-motivation on goneral activity and instrumental behavior. Psychological Review, 1968, 75, 1-22.

BROWN, J. S. The mottuation of behavior. New York: MoGraw-Hill, 1961.

EDMONSON, B. W., \& AMSEL, A. The effects of massing and distribution of extinction trials on the persistence of a fear-motivated in stru mental response. Journal of Companative \& Physiological Psychology, 1954, 47, 117-123.

HALL, J. F. Massed and spaced extinction in an avoidance conditioning situation. Journal of Genetic Psychology, 1955, 86, 179-182.

HOFFMAN, H. S., \& FLESHLER, M. A. A relay sequencing device for scrambling grid shock. Journal of the Experimental Analysis of Behavior, 1962, 5, 329-330.

MILLER, N. E. Learnable drives and rewards. In S. S. Stevens (Ed.), Handbook of experimental psychology. New York: Wiley, 1951. Pp. 435-472.

MURRAY, A. K., \& STRANDBERG, J. M. Development of a conditioned positive reinforcer through removal of an aversive stimulus. Journal of Comparative \& Physiological Psychology, 1965, 60, 281-283.

SEWARD, J. P. Drive, incentive, and reinforcement. Psychological Review, 1956, 63. 195-203.

SIEGEL, P. S., \& MILBY, J. B., JR. Secondary reinforcement in relation to shock termination-Second chapter. Psychological Bulletin, in press.

SPENCE, K. W. Behavior theory and conditioning New Haven: Yale University Press, 1956.

\title{
Comparison of the effect of two minor tranquilizers on escape behavior ${ }^{1}$
}

F. J. BREMNER, H. D. COBB, and T. J. NAGY, Trinity University, San Antonio, Tex. 78212

Four groups of Ss were dosed with different compounds while being tested on an impossible visual discrimination. It was found that the Ss treated with either of two minor tranquilizers (CDP or Serax) spent significantly less time on an escape platform than did Ss treated with tween-80 (the solvent for Serax) or water (the solvent for $C D P$ ).

In a series of studies conducted at Trinity University, it has been demonstrated that tranquilizer-dosed Ss, who have been submitted to a stress-producing situation (Bremner \& Trowill, 1962; Trowill, 1961), yield significant differences among group performances on a number of responses measured. Much of this data was reported by Bremner, Cobb, \& Hahn (1969), who showed that CDP-treated rats made more lever presses, discriminated better, and spent less time on an escape platform than did controls tested in the above-mentioned stress-producing situation.

The present paper reports the findings of a study comparing the results of two closely related minor tranquilizers, CDP and Serax, on the amount of time spent on an escape platform that has been taken to represent a measure of stress in the paradigm used.

\section{SUBJECTS}

Twenty-four naive, 90-day-old male Holtzman rats served as Ss. These Ss were randomly assigned to four groups of six Ss each. The groups were CDP-treated, 
Serax-treated, water-treated, and tween-80-treated.

\section{APPARATUS}

Four automatically programmed Skinner boxes, modified as reported by Bremner \& Trowill (1962), were used to test the above-described Ss. Briefly, the Skinner boxes were equipped with shock grids, stimulus-projector lights, and a combined manipulandum and food cup (referred to henceforth as the lever). The shock grid was wired to a scrambler from which the intensity and duration of the grid shock could be set. The scrambler was, in turn, wired to a programmer that controlled its onset. The lights were typical stimulus projectors, supplied by Lehigh Valley, that can be programmed to vary in 11 discrete stimulus-intensity steps. The lever was directly attached to a standard pellet dispenser so that a depression of the lever could place one $25-\mathrm{mg}$ food pellet in the hollow trough in the lever (Bremner \& Trowill, 1962).

In the right rear corner of the Skinner box at floor height, there was a plastic platf urm, $2.75 \times 2.66$ in. If the $S$ sat on this platform, it deactivated all aspects of the situation for as long as the $S$ remained. That is, the stimulus lights, the house light, the shock grid, and the food cup were all deactivated for the duration of the S's sitting on the platform. Since the use of this platform allowed the $S$ to withdraw from the situation, it was called the escape platform.

A series of electronic counters recorded not only the number of correct and incorrect lever presses, but also the number and duration of each use of the escape platform.

\section{PROCEDURE}

Upon receipt of a shipment of rats, they were randomly divided into groups, both as to drug treatment and also as to which of the four Skinner boxes they would be assigned to. They were then placed on a 22.5-h feeding schedule for 1 week. At the end of this week, the Ss were deprived of food for $48 \mathrm{~h}$ and then placed in the Skinner boxes on a CRF for food for $1 / 2 \mathrm{~h}$. Ss that did not make 80 unaided lever presses during this CRF training period were discarded. On the 2 nd day of training, the Ss were switched from a CRF schedule to a bright-dim discrimination problem in which the brightest stimulus from the stimulus projectors was food-rewarded, but the dimmest was unrewarded. On the 3rd day of training, the shock scrambler was turned on, with an intensity of $.015 \mathrm{~mA}$ and a duration of $.5 \mathrm{sec}$. The intensity of shock was increased gradually each day until it reached $.2 \mathrm{~mA}$ on the 15 th and final day of training. These training sessions lasted $45 \mathrm{~min}$, after which the $\mathrm{Ss}$

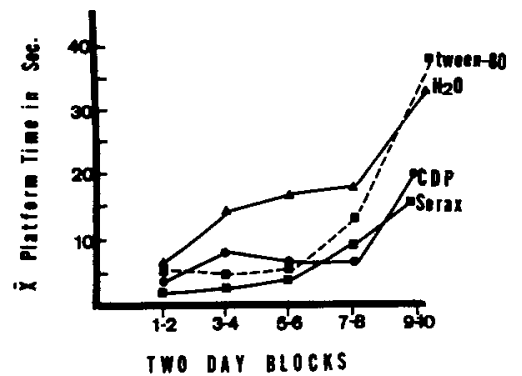

Fig. 1. Plot of mean platform time for the four groups over days of testing.

were given food in their home cages for $1 / 2 \mathrm{~h}$.

The testing procedure used was rather complex and will only be described briefly as it has been discussed in detail elsewhere (Bremner, Cobb, \& Hahn, 1969; Trowill, 1961; Nagy, 1969; Morris, 1969). In short, the $S$ s were given an impossible visual-discrimination problem, using the 11 programmable intensities of the stimulus projectors. The five brightest stimuli were rewarded with food upon the Ss' making a lever press, while the five dimmest stimuli yielded a grid shock upon the Ss' making a lever press. A lever press during the median-intensity stimulus was followed by shock $50 \%$ of the time and by food $50 \%$ of the time. These 11 stimuli were presented randomly during the 10 daily, 22-min testing sessions, so that there was an equal number of positive and negative stimuli presented. The final training shock intensity was again increased each day according to a fixed schedule, so that it was $1.0 \mathrm{~mA}$ on the 10 th and final day of testing (Nagy, 1969). The Ss were maintained on 22.5 -h food deprivation during the 10 days of testing, but were given food for $1 / 2 \mathrm{~h}$ in their home cages after each testing session. Simultaneously, with the beginning of testing, the Ss were orally dosed with the appropriate compound. These injections were administered $1 / 2 \mathrm{~h}$ before each session. The control Ss (tween-80 and water) were given an equal-part-by-volume injection. Water served as the solvent for CDP and tween-80 as the solvent for Serax.

\section{RESULTS}

All Ss were making at least two positive to one negative lever press at the end of the training session, and their platform time was low and irregular. In fact, while it was intended to test the Ss for differences on the last day of discrimination, the group means on Day 1 of testing were so close that it was not necessary to do so. Some indication of this can be seen in Fig. 1.

An analysis of variance was conducted on the escape platform time for each day for each group. The variables were days, groups, and Ss. This analysis showed that groups were significantly different from each other $(\mathrm{F}=3.6, \mathrm{p}<.05,3 / 20 \mathrm{df})$, and that days were significantly different $(F=9.3$, $\mathrm{p}<.001,9 / 180 \mathrm{df}$ ), but that Groups by Days was not significant. Figure 1 shows these effects. An orthogonal comparison was applied to the significant groups effect, yielding a significant difference $(F=7.0$, $\mathrm{p}<.025,1 / 20 \mathrm{df}$ ) between the two tranquilizer-treated groups (Serax and CDP) and the two control groups (water and tween-80). The residual was not significant $(F=2, p>.10,1 / 20 \mathrm{df})$.

\section{DISCUSSION}

The statistical treatments applied to the data of this study demonstrate that CDP- or Serax-dosed groups performed significantly differently from water- or tween-80-dosed groups (groups main effect, $p<.05$; and orthogonal comparison, $\mathrm{p}<.025$ ). Figure 1 shows that all groups increasingly use the platform over days (significant days effect, $\mathrm{p}<.001$ ), but that the tranquilizer-treated groups spend less time on the escape platform than do the control groups. Figure 1 also shows that tween- 80 has an apparent therapeutic effect, but that as shock increases over days, whatever effect there was is lost. It should be pointed out that this effect is not significant in any case (orthogonal comparison, $\mathrm{F}=2.0, \mathrm{df}=1 / 20$, $\mathrm{p}>$.10).

The above results can be easily explained if one will allow that platform time is a measure of stress, and that this stress is caused by the strong conflicting hunger drive and fear-producing electric shock. Furthermore, this conflict is compounded by the impossible visual discrimination. It would logically follow that the two minor tranquilizers, CDP and Serax, decrease the effect of the conflict, so that Ss attempt to escape the situation less of ten than do controls. In addition, it is of interest to note that these two biochemically similar compounds gave behaviorally similar results.

\section{REFERENCES}

BREMNER, F. J., COBB, H. D., \& HAHN, W. C. The effect of chlordiazepoxide on the behavior and brain serotonin levels of rats in a conflict situation. Psychopharmacologia, in press.

BREMNER, F. J., \& TROWILL, J. A. A combined manipulandum reinforcement arrangement. Journal of the Experimental Analysis of Behavior, 1962, 5, 339-340.

MORRIS, M. D. The effect of oxazepam in the conflict situation. Masters thesis, Trinity University, 1969.

NAGY, T. J. The effect of chlordiazepoxide on behavior and brain serotonin levels of rats in a conflict situation. Masters thesis, Trinity University, 1969.

TROWILL, J. A. An investigation of the drive properties of conflict in rats. Masters thesis, University of Massachu setts, 1961. NOTE

1. This study was supported in part by the Morrison Foundation, Grant No. MTRA*3. 